\title{
Efeito da Nicarbazina sobre o Desempenho de Frangos de Corte Recebendo Diferentes Níveis de Lisina Submetidos a Estresse por Calor
}

\author{
Juarez Lopez Donzele ${ }^{2}$, Rita Flávia Miranda de Oliveira ${ }^{2}$, Paulo Cegatto Cella ${ }^{4}$, \\ Adhemar Rodrigues de Oliveira Neto ${ }^{3}$
}

\begin{abstract}
RESUMO - Este trabalho foi realizado para avaliar o efeito da adição de nicarbazina e níveis de lisina na ração de frangos de corte no período de 1 a 21 dias de idade e seu efeito residual sobre o período de 22 a 49 dias de idade. As aves foram criadas em ambiente de alta temperatura. Foram utilizados 480 frangos de corte machos da marca Ross, em delineamento inteiramente casualizado em esquema fatorial 2x4 (adição ou não de nicarbazina e nível de lisina) seis repetições e 10 aves por unidade experimental. Foram utilizados dois programas no combate a coccidiose: um programa dual, em que as aves receberam nicarbazina de 1 a 21 dias e monensina de 22 a 49 dias, e um programa único de controle, no qual somente a monensina foi fornecida para as aves de 1 aos 42 dias. A ração e a água foram fornecidas à vontade. Verificou-se que as aves que receberam nicarbazina tiveram consumo de ração e ganho de peso menores e conversão alimentar pior do que as aves recebendo o programa controle. Os pesos corporais e rendimentos de carcaça e de cortes nobres foram reduzidos pelo efeito da nicarbazina. Os pesos absoluto e relativo de órgãos também foram reduzidos nas aves tratadas com nicarbazina, quando comparadas àquelas que não receberam a droga na ração. Não foi viável a inclusão de nicarbazina em rações para aves mantidas em ambiente de alta temperatura.
\end{abstract}

Palavras-chave: estresse de calor, frango de corte, lisina, nicarbazina

\section{Effect of Nicarbazin on the Performance of Broiler Chicks Fed Diets With Different Lysine Levels Submitted to Heat Stress}

\begin{abstract}
This work was conducted aiming to evaluate the effect of the addition nicarbazin and lysine levels in the diet of broilers from 1 to 21 days of age and its residual effect on the period from 22 to 49 days of age. The broilers were created in high environmental temperature. Four hundred and eighty male broilers Ross brand were used, in a completely randomized experimental design, in a $2 \times 4$ factorial arrangement (addition or not of nicarbazin and level of lysine), six replications of 10 birds per experimental unit. Nicarbazin and monensin were added to $1-21 \mathrm{~d}$ and 22-42d diets, respectively, in a dual anticoccidial program and a control anticoccidial program where only the monensin was fed to the birds from 1 to $42 \mathrm{~d}$. The diet and the water were ad libitum fed. Birds fed nicarbazin-monenzin showed lower feed intake and average weight gain, and higher feed:gain ratio than those fed control anticoccidial program. The body weights and the carcass and edible cuts yield were reduced by the effect of the nicarbazin. The birds fed diet with nicarbazin showed reduced absolute and relative organs weights as compared to those that did not receive the drug in the diet. The addition of nicarbazin in diets for broilers under heat stress is not feasible.
\end{abstract}

Key Words: broiler, heat stress, lysine, nicarbazin

\section{Introdução}

A presença de coccidiose em lotes de frangos de corte é constante na indústria avícola (SHIRLEY, 1994), acarretando depressão de desempenho, e, consequentemente, aumento do custo de produção. Segundo CASTRO (1994), a coccidiose, clínica e subclínica, onera os custos de produção em cerca de 500 milhões de dólares em todo o mundo.

Na tentativa de minimizar tais prejuízos, vários produtos são lançados no mercado mundial com vista ao combate da coccidiose. Os anticoccidianos utilizados na indústria avícola, químicos (nicarbazina, robenidina, dinitolmida, halofuginona, diclazuril) e ionóforos (monensina, narasim, salinomicina, lasalocida), podem ser usados isoladamente ou combinados nas rações e nas diferentes fases de vida das aves.

A nicarbazina, um coccidiostático sintetizado quimicamente em laboratório, foi introduzido na Inglaterra em 1955, como eficiente coccidiostático no combate a Eimeria tenella dos cecos de frangos de corte. Os primeiros casos de resistência foram descritos em 1964(CHAPMAN, 1998). Sugere-se que anicarbazina (4-4-Dinitrocarbanilida (DNC) 2- Hidroxi-4,6Dimmetil Pirimidina) atue sobre o metabolismo da eiméria por meio da inibição do $\mathrm{NAD}^{+}$, em que os

\footnotetext{
1 Projeto financiado pela FAPEMIG.

2 Professor do DZO/UFV.

${ }^{3}$ Estudante de Doutorado, DZO/UFV - CEP: 36570-000.

${ }^{4}$ Estudante de Doutorado, DZO/UEM.
} 
esquizontes de segunda geração e seus merozoítos são destruídos ou têm seu desenvolvimento interrompido (PLANALQUÍMICA,1994).

Embora a nicarbazina seja eficiente no combate às eimérias, suautilização em programas visando o controle de coccidiose em frangos de corte deve ser cauteloso, uma vez que vários autores constataram aumento na mortalidade (KESHAVARZe McDOUGALD, 1981; McDOUGALD e McQUISTION, 1980; SAMMELWITZ, 1965) e, piora no ganho de peso e conversão alimentar, além de redução no consumo de ração e de água em frangos de corte tratados com esta droga (CUCKLER et al., 1956; BARTOV, 1989).

Os efeitos da nicarbazina sobre as atividades fisiológicas das aves não são totalmente compreendidos, contudo, sabe-se que o seu uso estimula o aumento no débito cardíaco e a produção de calor corporal, com aumento na temperatura retal. Ocorre redução da habilidade dos frangos de corte de manter sua homeotermia, já que a nicarbazina também reduz a taxa respiratória das aves (WIERNUSZ e TEETER, 1995; BEERS et al., 1989), influenciando o principal mecanismo de perda de calor corporal das aves, quando mantidas em temperatura elevada.

Este trabalho foi conduzido com objetivo de avaliar os efeitos da nicarbazina sobre o desempenho de frangos de corte recebendo diferentes níveis de lisina, mantidos em ambiente de alta temperatura.

\section{Material e Métodos}

Foram utilizados 480 frangos de corte machos da marca Ross, com peso inicial médio de $43 \mathrm{~g}$, em delineamento experimental inteiramente casualizado em arranjo fatorial $2 \times 4$ (com ou sem nicarbazina, e quatro níveis de lisina) com seis repetições de 10 aves por unidade experimental, mantidas sob estresse de calor. Os níveis de lisina foram, 1,00,1,10,1,20 e 1,30\% para a fase inicial (1 a 21 dias) , 0,90, 1,0, 1,10 e 1,20\% nas rações para a fase de crescimento (22 a 42 dias) e $0,8,0,9,1,0$ e $1,1 \%$ nas rações para a fase final ( 23 a 49 dias) das aves.

As aves tratadas com nicarbazina, receberam 120 ppm desta droga na ração no período de 1 a 21 dias de idade, enquanto que nas demais fases (crescimento e final) foram tratadas com um ionóforo (monensina), constituindo um programa coccidiostático dual. No tratamento sem adição de nicarbazina, utilizou-se somente monensina, na ração, durante todo o período experimental (1 a 49 dias). Os aminoácidos L-treonina, L-isoleucina, L-arginina e L-triptofano foram suplementados para estabeleceropadrãode proteínaideal propostopor BAKER e HANN (1994). A ração experimental (Tabela 1) foi
Tabela 1 - Composição centesimal das rações basais de acordo com as fases de crescimento

Table 1 - Percentage composition of basal diets according to the growth phases

\begin{tabular}{|c|c|c|c|}
\hline \multirow[b]{2}{*}{$\begin{array}{l}\text { Ingrediente } \\
\text { Ingredient }\end{array}$} & \multicolumn{3}{|c|}{$\begin{array}{c}\text { Fase de crescimento } \\
\text { Growth phase }\end{array}$} \\
\hline & $\begin{array}{l}1-21 \text { dias } \\
1-21 \text { days }\end{array}$ & $\begin{array}{c}22-42 \text { dias } \\
22-42 \text { days }\end{array}$ & $\begin{array}{r}43-49 \text { dias } \\
43-49 \text { days }\end{array}$ \\
\hline Milho & 60,18 & 66,06 & 71,40 \\
\hline Corn & & & \\
\hline $\begin{array}{l}\text { Farelo de soja, } 46 \% \mathrm{~PB} \\
\text { Soybean meal, } 46 C P\end{array}$ & 327,00 & 21,00 & 15,00 \\
\hline Glutenose 60 & 7,00 & 7,00 & 7,00 \\
\hline $\begin{array}{l}\text { Glutenose } 60 \\
\text { F. bicálcico } \\
\text { Dicalcium phosphate }\end{array}$ & 1,90 & 1,70 & 1,60 \\
\hline $\begin{array}{l}\text { Calcário } \\
\text { Limestone }\end{array}$ & 1,10 & 1,20 & 1,10 \\
\hline $\begin{array}{l}\text { Caulim } \\
\text { Caulin }\end{array}$ & 0,41 & 0,48 & 0,57 \\
\hline $\begin{array}{l}\text { Bicarbonato de sódio } \\
\text { Sodium bicarbonate }\end{array}$ & 0,10 & 0,10 & 0,10 \\
\hline $\begin{array}{l}\text { Sal } \\
\text { Salt }\end{array}$ & 0,40 & 0,40 & 0,40 \\
\hline $\begin{array}{l}\text { Óleo de soja } \\
\text { Soybean oil }\end{array}$ & 1,00 & 1,00 & 2,00 \\
\hline $\begin{array}{l}\text { Mistura vitamínica }{ }^{1} \\
\text { Vitamin mix }\end{array}$ & 0,50 & 0,50 & 0,30 \\
\hline $\begin{array}{l}\text { Mistura mineral } \\
\text { Mineral mix }\end{array}$ & 0,05 & 0,05 & 0,05 \\
\hline $\begin{array}{l}\text { Promocobre Violeta } \\
\text { Violet promocopper }\end{array}$ & 0,10 & 0,10 & - \\
\hline $\begin{array}{l}\text { L-lisina } \\
\text { L-lysine }\end{array}$ & 0,16 & 0,20 & 0,24 \\
\hline $\begin{array}{l}\text { DL-metionina } \\
\text { DL-methionine }\end{array}$ & 0,03 & 0,05 & 0,02 \\
\hline $\begin{array}{l}\text { L-arginina } \\
\text { L-arginine }\end{array}$ & 0,07 & - & - \\
\hline $\begin{array}{l}\text { L-treonina } \\
\text { L-treonine }\end{array}$ & - & 0,04 & 0,04 \\
\hline $\begin{array}{l}\text { L-triptofano } \\
\text { L-triptophan }\end{array}$ & - & 0,12 & 0,16 \\
\hline $\begin{array}{l}\text { L-isoleucina } \\
\text { L-isoleucine } \\
\text { Composição calculada } \\
\text { Calculated composition }\end{array}$ & $a^{3}$ & - & 0,02 \\
\hline $\begin{array}{l}\text { Calculated composition } \\
\text { Proteína bruta (\%) } \\
\text { Crude protein }\end{array}$ & 21,69 & 19,55 & 17,29 \\
\hline $\begin{array}{l}\text { Lisina total }(\%) \\
\text { Total lysine }\end{array}$ & 1,00 & 0,90 & 0,80 \\
\hline $\mathrm{EM}(M E)(\mathrm{kcal} / \mathrm{kg})$ & 3.000 & 3.100 & 3.150 \\
\hline $\begin{array}{l}\text { Cálcio } \\
\text { Calcium }\end{array}$ & 0,995 & 0,918 & 0,877 \\
\hline $\begin{array}{l}\text { Fósforo disponível } \\
\text { Available phosphorus }\end{array}$ & 0,453 & 0,387 & 0,344 \\
\hline
\end{tabular}

1 Conteúdo por kg de ração (Content per kg of diet): Vit. A - 15.000.000 UI; Vit $D_{3}-1.500 .000$ UI; Vit. E - 15.000 UI; Vit. $B_{1}-2,0$ g; Vit. $B_{2}-4,0 \mathrm{~g}$; Vit. $\mathrm{B}_{6}-3,0 \mathrm{~g}$; Vit. $\mathrm{B}_{12}-0,015 \mathrm{~g}$; ácido nicotínico (nicotinic acido) - $25 \mathrm{~g}$; ácido pantotênico (panthothenic acido) - $10 \mathrm{~g}$; Vit. $\mathrm{K}_{3}-3,0 \mathrm{~g}$; ácido fólico (pholic acid) - 1,0 g; selênio (selenium) - $250 \mathrm{mg}$; antioxidante (antioxidant) BHT - $10 \mathrm{~g}$; e veículo (vehicle) q.s.p. - $1000 \mathrm{~g}$.

2 Conteúdo porkg de ração (Contentperkg ofdiet): manganês (manganese) - $80 \mathrm{~g}$; ferro (iron) - $80 \mathrm{~g}$; zinco (zinc) - $50 \mathrm{~g}$; cobre (copper) - $10 \mathrm{~g}$; cobalto (cobalt) - $2 \mathrm{~g}$; iodo (iodine) - $1 \mathrm{~g}$; cloreto de colina (coline cloret) $(50 \%)$

- $6 \mathrm{~g}$; e veículo (vehicle) q.s.p. - $500 \mathrm{~g}$.

3 Valores calculados segundo ROSTAGNO et al., 1992 (Calculated values according to ROSTAGNO et al., 1992). 
1054 Rev. bras. zootec.

formulada segundo recomendação de ROSTAGNO et al. (1992) e, assim como a água, foi fornecida à vontade.

As aves foram alojadas em baterias metálicas com 12 compartimentos de 0,85 x $0,85 \mathrm{~m}$ cada, dispostas em câmaras climáticas com temperatura e umidade relativa do ar controladas. O programa de luz foi contínuo (24 horas de luz artificial) durante todo o período experimental, utilizando-se duas lâmpadas fluorescentes de 75 watts, por câmara.

As aves e as rações experimentais foram pesadas no início e no final de cada período experimental para determinação do ganho de peso, do consumo de ração e para cálculo posterior da conversão alimentar. A mortalidade das aves, em cada período experimental, também foi registrada.

No 49o dia de idade, 12 aves de cada tratamento foram abatidas para avaliação do peso absoluto e dos respectivos rendimentos da carcaça, cortes nobres (coxa, sobrecoxa, peito com e sem osso), órgãos comestíveis (moela, fígado e coração).

As análises estatísticas das variáveis de desempenho, rendimento de cortes nobres e peso de órgãos foram realizadas utilizando-se o programa SAEG (Sistema para Análises Estatísticas e Genéticas), desenvolvido na UNIVERSIDADE FEDERAL DE VIÇOSA - UFV (1982).

\section{Resultados e Discussão}

As temperaturas e umidade relativas das salas foram registradas, diariamente, durante o período experimental, três vezes ao dia, por meio de termômetros de bulbo seco e úmido, de globo negro e de máxima e mínima, mantidos no centro da sala, representando o ambiente dos animais (Tabela 2).

$\mathrm{O}$ efeito da nicarbazina sobre o desempenho de frangos de corte no período de 1 a 21 dias de idade, recebendo diferentes níveis de lisina na ração, são mostrados na Tabela 3.

Não ocorreu interação entre a adição ou não de nicarbazina e os níveis de lisina para todas as variáveis estudadas. De forma similar ao ocorrido com o nível de lisina neste trabalho, BARTOV (1989) não observaram interação entre a nicarbazina e os níveis de proteína bruta da ração.

Os níveis de lisina não influenciaram o consumo de ração (CR) de frangos de corte no período de 1 a 21 dias de idade. Por outro lado, o ganho de peso (GP) e a conversão alimentar (CA), independente da nicarbazina, foram melhorados de forma linear $(\mathrm{P}<0,01)$ à medida que se aumentou o nível de lisina da ração. Apesar de ter ocorrido melhora linear, não se constatou variação na CA em valor absoluto a partir do nível de $1,20 \%$ de lisina.

Com relação a adição da nicarbazina na ração verificou-se que as aves que receberam os tratamentos contendo nicarbazina apresentaram menor $(\mathrm{P}<0,01) \mathrm{CR}$ e que este não se alterou em razão da variação no nível de lisina da ração. Estes resultados corroboram aqueles obtidos por KESHAVARZ e MADOUGALD (1982) e BARTOV (1989), que também verificaram efeito negativo da nicarbazina sobre o consumo de ração das aves submetidas a temperaturas elevadas.

Considerando que em condições de alta temperatura as aves diminuem o consumo na tentativa de reduzir a produção de calor metabólico (OLIVEIRA NETO, 1999), o efeito negativo da nicarbazina sobre o CR nestas condições pode ser explicado pela sua ação de aumentar a taxa metabólica e produção de calor corporal das aves. De acordo com resultados de BEERS et al. (1989) e WIERNUZ e TEETER (1995) frangos de corte submetidos a estresse de calor tiveram sua taxa metabólica e produção de calor aumentadas em razão da nicarbazina incorporada às rações. Portanto, a diminuição do $\mathrm{CR}$ das aves recebendo nicarbazina constitui mecanismo de defesa para evitar o aumento na quantidade de calor metabólico que teria de ser dissipado para o meio ambiente.

Verificou-se efeito da nicarbazina $(\mathrm{P}<0,01)$ sobre o GP que reduziu em $16,9 \%$. Considerando que o consumo de ração diminuiu em $9,3 \%$, podese inferir que a variação no GP ocorreu não somente em razão daquela verificada no CR, mas também devido a perda de eficiência de utilização do alimento. Este efeito ficou evidenciado quando se constatou aumento $(\mathrm{P}<0,01)$ de $10 \%$ na $\mathrm{CA}$ das aves alimentadas com ração contendo nicarbazina. Os resultados obtidos neste trabalho estão coerentes com os de BARTOV (1989) que também verificaram efeito negativo da nicarbazina sobre o GP e a CA das aves.

Com base nos efeitos da nicarbazina sobre as características de desempenho, calculou-se que a redução no $\mathrm{CR}$ e o aumento da $\mathrm{CA}$, podem explicar, respectivamente, 53,3 e 46,7\% da redução do GP das aves.

Apesar de não ter ocorrido interação, observouse que menores valores absolutos de GP e maior de CA foram obtidos no nível mais alto de lisina $(1,3 \%)$. 
DONZELE et al.

Tabela 2 - Condições ambientais observadas nas câmaras climáticas durante cada período experimental Table 2 - Average environmental conditions observed in climatic chambers during each experimental period

\begin{tabular}{|c|c|c|c|c|}
\hline $\begin{array}{l}\text { Idade (dias) } \\
\text { Age (days) }\end{array}$ & $\operatorname{Tar}^{1}\left({ }^{\circ} \mathrm{C}\right)$ & $\mathrm{UR}^{2}(\%)$ & $\operatorname{Tgn}^{3}\left({ }^{\circ} \mathrm{C}\right)$ & $\begin{array}{l}\text { ITGU }^{4} \\
\text { BGHI }\end{array}$ \\
\hline \multicolumn{5}{|c|}{$\begin{array}{l}\text { Com nicarbazina } \\
\text { With nicarbazin }\end{array}$} \\
\hline $\begin{array}{l}1-21 \\
22-42 \\
43-49 \\
\end{array}$ & $\begin{array}{l}35,0 \pm 0,11 \\
35,0 \pm 0,17 \\
35,0 \pm 0,14\end{array}$ & $\begin{array}{l}42,9 \pm 1,56 \\
42,3 \pm 1,01 \\
42,9 \pm 1,90 \\
\end{array}$ & $\begin{array}{c}34,0 \pm, 05 \\
34,2 \pm 0,29 \\
34,2 \pm 0,26\end{array}$ & $\begin{array}{l}82,7 \pm 0,26 \\
82,8 \pm 0,35 \\
83,0 \pm 0,35 \\
\end{array}$ \\
\hline \multicolumn{5}{|c|}{$\begin{array}{l}\text { Sem nicarbazina } \\
\text { Without nicarbazin }\end{array}$} \\
\hline $\begin{array}{l}1-21 \\
22-42 \\
43-49\end{array}$ & $\begin{array}{l}33,5 \pm 0,58 \\
35,0 \pm 0,17 \\
35,0 \pm 0,14\end{array}$ & $\begin{array}{l}43,1 \pm 3,39 \\
42,2 \pm 0,91 \\
43,1 \pm 1,96 \\
\end{array}$ & $\begin{array}{l}33,2 \pm 0,50 \\
34,2 \pm 0,29 \\
34,2 \pm 0,26\end{array}$ & $\begin{array}{l}82,0 \pm 0,59 \\
83,5 \pm 0,33 \\
83,5 \pm 0,29 \\
\end{array}$ \\
\hline
\end{tabular}

Temperatura do ar (Air temperature); 2 Umidade relativa (Relative humidity)
Temperatura de Globo e Umidade (Globe Temperature and Humidity Index).

Tabela 3 - Efeito da nicarbazina sobre o desempenho de frangos de corte no período de 1 a 21 dias de idade, mantidos em estresse de calor

Table 3 - Effect of nicarbazin on performance of broilers under heat stress from 1 to $21 d$-old

\begin{tabular}{|c|c|c|c|c|c|c|c|c|c|c|}
\hline \multirow{3}{*}{$\begin{array}{l}\text { Característica } \\
\text { Characteristic }\end{array}$} & \multicolumn{4}{|c|}{$\begin{array}{l}\text { Sem nicarbazina } \\
\text { Without nicarbazin }\end{array}$} & & \multicolumn{4}{|c|}{$\begin{array}{l}\text { Com nicarbazina } \\
\text { With nicarbazin }\end{array}$} & \multirow[b]{3}{*}{$\begin{array}{c}\text { Média } \\
\text { Mean }\end{array}$} \\
\hline & \multicolumn{4}{|c|}{$\begin{array}{c}\text { Nível de lisina }(\%) \\
\text { Lysine level }\end{array}$} & & \multicolumn{4}{|c|}{$\begin{array}{c}\text { Nível de lisina (\%) } \\
\text { Lysine level }\end{array}$} & \\
\hline & 1,0 & 1,1 & 1,2 & 1,3 & $\begin{array}{l}\text { Média } \\
\text { Mean }\end{array}$ & 1,0 & 1,1 & 1,2 & 1,3 & \\
\hline$\overline{\mathrm{CR}(F I)(g)}$ & 832 & 829 & 816 & 836 & $829^{\mathrm{A}}$ & 733 & 770 & 736 & 769 & $752^{\mathrm{B}}$ \\
\hline $\mathrm{GP}(W G)(g)$ & $615^{1}$ & 637 & 650 & 641 & $635^{\mathrm{A}}$ & $507^{1}$ & 516 & 539 & 556 & $528^{B}$ \\
\hline $\mathrm{CA}(F / G)$ & $1,34^{1}$ & 1,30 & 1,25 & 1,30 & $1,30^{\mathrm{B}}$ & $1,44^{1}$ & 1,49 & 1,37 & 1,38 & $1,43^{\mathrm{A}}$ \\
\hline MORT, $1 / 1$ & - & - & - & - & $1,25^{\mathrm{B}}$ & - & - & - & - & $9,57^{\mathrm{A}}$ \\
\hline
\end{tabular}

${ }^{1}$ Efeito linear (Linear effect) $(\mathrm{P}<0,01)$

Médias seguidas por letras diferentes na mesma linha diferem pelo teste $T(P<0,05)$ (Means followed by different letters within a row differ by $T$ test [P.05]). CR - consumo de ração (FI - feed intake); GP - ganho de peso (WG - weight gain); CA - conversão alimentar (F:G - feed:gain ratio); MORT - mortalidade (MORT - mortality).

Estes resultados contrastam aqueles obtidos por BARTOV (1989), que, avaliando a ação da nicarbazina nas rações de frangos de corte, constatou que seus efeitos negativos sobre o GP e a eficiência alimentar foram independentes do nível de proteína das rações.

Além do desempenho, a taxa de mortalidade também foi influenciada $(\mathrm{P}<0,01)$ pela nicarbazina, aumentando de 1,25 para $9,57 \%$. Diferentes pesquisadores, entre eles MAcDOUGALD e McQUISTION (1980) e KESHAVARZ e MACDOUGALD (1981), também verificaram maior mortalidade de frangos de corte em razão da presença de nicarbazina na ração. O alto índice de mortalidade dos frangos tratados com nicarbazina pode estar associado ao possível aumento da taxa metabólica e da produção de calor, levando a ave a um estado de hipertermia não compensada, culminando com a morte. Segundo relato de Fany (1965), citado por MAcDOUGALD e MACQUISTION (1980), frangos de corte recebendo nicarbazina apresentam maior taxa metabólica e menor resistência a alta temperatura ambiente, desenvolvendo hipertermia mais rapidamente que os não tratados. 
1056 Rev. bras. zootec.

O efeito residual da nicarbazina sobre o desempenho de frangos de corte no período de 22 a 42 dias de idade e o desempenho das aves no período total (1 a 49 dias de idade) são mostrados na Tabela 4. A utilização da nicarbazina na ração de frangos de corte na fase inicial (1 a 21 dias) teve efeito residual sobre o desempenho das aves nas fases de crescimento e final ( 22 a 49 dias) diminuindo $(\mathrm{P}<0,01)$ o GP e CR e aumentando a taxa de mortalidade de $2,5 \%$ para $16,96 \%$, sem, no entanto, influenciar $(\mathrm{P}<0,05)$ a CA.

A nicarbazina fornecida na fase inicial aos frangos de corte também refletiu negativamente $(\mathrm{P}<0,01)$ sobre todos as características de desempenho avaliadas e sobre a taxa de mortalidade das aves, quando se considerou o período total de 1 a 49 dias de idade.

Pelos resultados obtidos pode-se deduzir que o comprometimento do GP dos frangos nas fases de crescimento e final e, consequentemente, no período total, ocorreu em razão da incapacidade das aves de compensarem o reduzido CR da fase inicial.
Observou-se que a adição de nicarbazina na ração de frangos de corte na fase inicial (1 a 21 dias) influenciou negativamente os pesos, absoluto e relativo, do fígado, moela e coração, que foram menores $(\mathrm{P}<0,01)$ aos 49 dias (Tabela 5). A redução no CR e, consequentemente, notamanho de órgãos metabolicamente ativos, provavelmente constituiu em ajuste fisiológico das aves na tentativa de compensar o aumento na produção de calor metabólico provocado por ação da nicarbazina.

Os pesos, absoluto e relativo, da carcaça e dos cortes nobres de frangos de corte mantidos em ambiente de alta temperatura foram influenciados $(\mathrm{P}<0,01)$ pela nicarbazina (Tabela 6). Os pesos, absoluto e relativo, da carcaça e dos corte nobres, com exceção do peso relativo da carcaça aos 49 dias, foram menores $(\mathrm{P}<0,01)$ nos frangos de corte que receberam nicarbazina na fase inicial. $O$ maior peso relativo $(\mathrm{P}<0,01)$ da carcaça das aves que receberam nicarbazina pode ser explicado pelos menores pesos relativos dos órgãos avaliados.

Tabela 4 - Efeito da nicarbazina sobre o desempenho de frangos de corte no período de 22 a 49 e 1 a 49 dias de idade, mantidos em estresse de calor

Table 4 - Effect of nicarbazin on the performance of broilers under heat stress from 22 to 49 and 1 to $49 \mathrm{~d}$-old

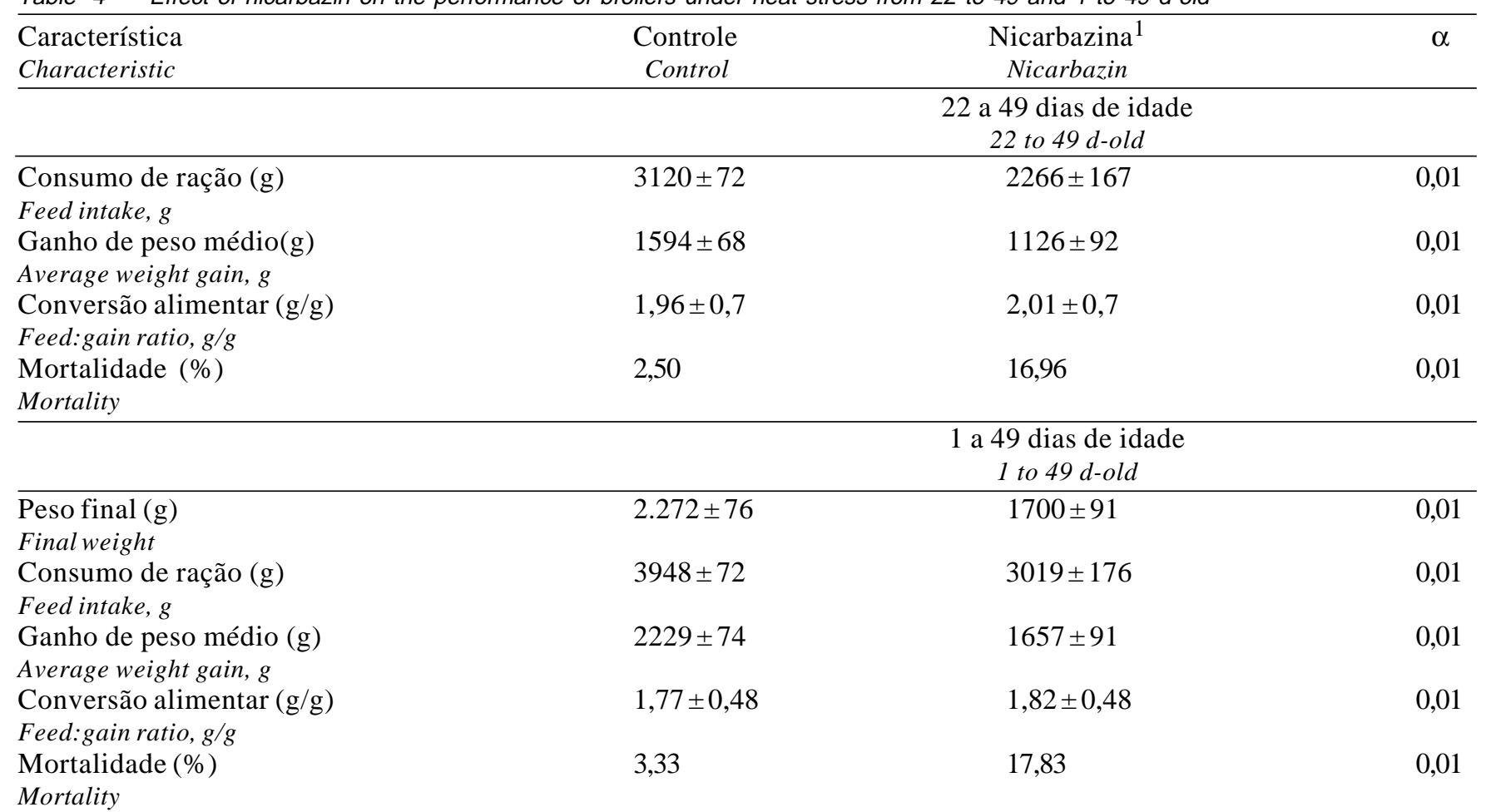

\footnotetext{
$\overline{1}$ A adição de nicarbazina foi feita somente na ração inicial (1 a 21 dias de idade) (The nicarbazin was included only in the initial ration [1 to 21 days of age]).
} 
Tabela 5 - Efeito da nicarbazina sobre os pesos, absoluto e relativo, de vísceras de frangos de corte aos 49 dias de idade, mantidos em estresse de calor Table 5 - Effect of nicarbazin on the absolute and relative weights of vicera of broilers at $49 d$-old under heat stress

\begin{tabular}{lccc}
\hline Característica & Controle & $\begin{array}{c}\text { Nicarbazina } \\
\text { Control }\end{array}$ & $\alpha$ \\
\cline { 3 - 3 } & & $\begin{array}{c}\text { Peso absorbazin } \\
\text { Absolute weight }\end{array}$ & \\
\hline $\begin{array}{l}\text { Moela } \\
\text { Gizzard }\end{array}$ & $24,8 \pm 1,85$ & $17,0 \pm 1,30$ & 0,01 \\
$\begin{array}{l}\text { Coração } \\
\text { Heart } \\
\text { Fígado } \\
\text { Liver }\end{array}$ & $8,7 \pm 0,74$ & $5,4 \pm 0,53$ & 0,01 \\
\hline & $33,9 \pm 3,69$ & $21,7 \pm 2,05$ & 0,01 \\
\hline $\begin{array}{l}\text { Moela } \\
\text { Gizzard } \\
\text { Coração }\end{array}$ & $1,30 \pm 0,11$ & $1,16 \pm 0,59$ & 0,01 \\
$\begin{array}{l}\text { Heart } \\
\text { Fígado }\end{array}$ & $0,45 \pm 0,36$ & $0,37 \pm 0,35$ & 0,01 \\
Liver & & & \\
\hline
\end{tabular}

Tabela 6 - Efeito da nicarbazina sobre o rendimento de carcaça e de cortes nobres de frangos de corte aos 49 dias de idade, mantidos em estresse de calor

Table 6 - Effect of nicarbazin on the carcass yield and edible parts of broilers at $49 d$-old, under heat stress

\begin{tabular}{|c|c|c|c|}
\hline \multirow[t]{2}{*}{$\begin{array}{l}\text { Característica } \\
\text { Characteristic }\end{array}$} & \multirow[t]{2}{*}{$\begin{array}{c}\text { Controle } \\
\text { Control }\end{array}$} & $\begin{array}{c}\text { Nicarbazina }^{1} \\
\text { Nicarbazin }\end{array}$ & \multirow[t]{2}{*}{$\alpha$} \\
\hline & & $\begin{array}{c}\text { Peso absoluto }(\mathrm{g}) \\
\text { Absolute weight }\end{array}$ & \\
\hline Carcaça & $1914 \pm 66,54$ & $1463 \pm 85,96$ & 0,01 \\
\hline Carcass & & & \\
\hline $\begin{array}{l}\text { Coxa } \\
\text { Drumstick }\end{array}$ & $261 \pm 8,81$ & $188 \pm 12,35$ & 0,01 \\
\hline $\begin{array}{l}\text { Sobrecoxa } \\
\text { Thigh }\end{array}$ & $268 \pm 14,73$ & $193 \pm 10,08$ & 0,01 \\
\hline $\begin{array}{l}\text { Peito com osso } \\
\text { Breast with bone }\end{array}$ & $511 \pm 30,16$ & $367 \pm 21,09$ & 0,01 \\
\hline $\begin{array}{l}\text { Peito sem osso } \\
\text { Breast with bone }\end{array}$ & $349 \pm 26,88$ & $135 \pm 10,17$ & 0,01 \\
\hline
\end{tabular}

Peso relativo $(\%)$

\begin{tabular}{lccc} 
& & \multicolumn{2}{c}{ Relative weight } \\
\hline $\begin{array}{l}\text { Carcaça } \\
\begin{array}{l}\text { Carcass } \\
\text { Coxa }\end{array}\end{array}$ & $85,02 \pm 1,39$ & $86,54 \pm 1,18$ & 0,01 \\
$\begin{array}{l}\text { Drumstick } \\
\text { Sobrecoxa }\end{array}$ & $13,65 \pm 0,39$ & $12,79 \pm 0,38$ & 0,01 \\
$\begin{array}{l}\text { Thigh } \\
\text { Peito com osso } \\
\text { Breast with bone }\end{array}$ & $26,67 \pm 0,96$ & $24,68 \pm 0,65$ & 0,01 \\
$\begin{array}{l}\text { Peito sem osso } \\
\text { Breast with bone }\end{array}$ & $18,21 \pm 0,95$ & $9,11 \pm 0,38$ & 0,01 \\
\hline
\end{tabular}

\section{Conclusões}

A adição de nicarbazina em ração inicial para frangos de corte, criados em condições de alta temperatura, independente do nível de lisina da ração, piora o desempenho e aumenta a taxa de mortalidade nas fases inicial, de crescimento e final e reduz o rendimento dos corte nobres e o peso dos órgãos das aves aos 49 dias de idade.

\section{Referências Bibliográficas}

BAKER, D.H., HAN, Y. 1994. Ideal amino acid profile for chicks during the first three weeks posthatching. Poult. Sci., 73:1441-1447.

BARTOV, I. 1989. Lack of effect of dietary factors on nicarbazin toxicity in broiler chicks. Poult. Sci., 68:145-152.

BEERS, K.W., RAUP, T.J., BOTTJE, W.G. et al. 1989. Physiological responses of heat stress broilers fed nicarbazin. Poult. Sci., 68:428-434.

CASTRO, A.G.M. Situação atual da coccidiose no Brasil importância econômica. In: SIMPÓSIO INTERNACIONAL SOBRE COCCIDIOSE, 1994, Santos, SP. Anais... Santos, SP: FACTA, 1994. p.45-54.

CHAPMAN, H.D. Anticoccidial agents for poultry. In: Aditivos na produção de ruminantes, aditivos na produção de não ruminantes e fronteiras do melhoramento genético animal. REUNIÃO ANUAL DA SOCIEDADE BRASILEIRA DE ZOOTECNIA, 35, Botucatu, SP. Anais... Botucatu, SP: SBZ, 1998. p. 219-236.

CUCKLER, A.C., MALANGA, C.M., OTT, W.H. 1956. The antiparasitic activity of nicarbazin. Poult. Sci., 68:145-152.

KESHAVARZ, K., McDOULGALD, L.R. 1981. Influenca of anticoccidial drugs on losses of broiler chickens from heat stress and coccidiosis. Poult. Sci., 60:2423-2428.

McDOUGALD, L.R., McQUISTION, T.E. 1980. Motality from heat stress in broiler chickens influenced by anticoccidial drugs. Poult. Sci., 59:2421-2423.

OLIVEIRA NETO, A.R. Efeito de níveis de energia da ração e da temperatura ambiente sobre o desempenho e os parâmetros fisiológicos de frangos de corte. Viçosa, MG: UFV, 1999. 111 p. Dissertação (Mestrado em Zootecnia) - Universidade Federal de Viçosa, 1999.

PLANALQUÍMICA: anticoccidianos. In: SIMPÓSIO INTERNACIONAL SOBRE COCCIDIOSE, 1994, Santos, SP. Anais... Santos, SP: FACTA, 1994. p.159-166.

ROSTANGO, H.S., SILVA, D.J. COSTA, P.M.A. et al. 1992. Composição de alimentos e exigências nutricionais de aves e suínos (Tabelas brasileiras). Viçosa, MG: UFV. 59p.

SAMMELWITZ, P.H. 1965. Heat stress mortality in broilers. Poult. Sci., 44:1412 (abstr.).

SHIRLEY, M.W. Epizootiologia. In: SIMPÓSIO INTERNACIONAL SOBRE COCCIDIOSE, 1994, Santos, SP. 1994. Anais... Santos, SP: FACTA, 1994. p.11-22.

UNIVERSIDADE FEDERAL DE VIÇOSA - UFV. 1982. Manual de utilização do programa SAEG (Sistemas para Análises Estatísticas e Genéticas). Viçosa-MG: UFV. 59p.

WIERNUZ, C.J., TEETER, R.G. 1995. Nicarbazin effects on broiler thermobalance during high ambient temperature stress. Poult. Sci., 74:577-580.

Recebido em: 24/05/00 Aceito em: 23/01/01 